\title{
COVID and CopMich: comparing and contrasting COVID-19 experiences in the USA and Scandinavia
}

\section{Juan J. Andino, James M. Dupree (D, Christian F. S. Jensen, Ganesh S. Palapattu, Jens Sønksen and Daniela Wittmann (D)}

On 11 March 2020, the World Health Organization declared SARS-CoV-2 and its associated disease, COVID-19, a global pandemic. Across the world, governments took action to slow the spread and hospitals rushed to accommodate an influx of patients with this highly infectious and lethal disease. The urology departments in Ann Arbor, Michigan, USA, and Herlev and Gentofte, Copenhagen, Denmark which are linked by the pre-existing CopMich Collaborative - had to respond with massive changes to the organization, staffing and workload of their teams. In this Viewpoint, authors from different urological subspecialties and at different career stages reflect on their experiences during the pandemic. Although their countries' responses to the COVID-19 pandemic differed radically, the similarities between the responses in Copenhagen and Michigan demonstrate the universal characteristics of medicine and the value of teamwork, flexibility and collaboration.

\section{0] What were the immediate changes in your institution in response to COVID-19?}

Ganesh S. Palapattu. COVID-19 has proven to be a crisis like no other. When it became clear that the pandemic was headed our way, our health system rapidly strategized and deployed a series of measures to monitor and mitigate the oncoming health-care disaster. Almost immediately a command centre was created, composed of a small multidisciplinary group of individuals spanning clinical operations, infectious disease, epidemiology, supply chain, critical care and communications, among others. This group met daily during our peak, often twice a day, to assess the current status, plan for the immediate future and identify and solve problems. This group was also key in providing timely and informative updates to our health system and community. At the same time, a team of Michigan Medicine experts, alongside collaborators from around the country, developed Michigan-specific prediction models and liaised with adjoining health systems and local and state government and public health departments. Furthermore, space allocations were quickly re-arranged in the span of days to create a regional infectious containment unit (RICU) a negative pressure unit comprising a substantial number of isolation rooms equipped with critical care medical equipment in compliance with CDC guidelines. As many of you know, the Detroit area was hit hard by COVID-19. I am proud to say that our health-care system responded to the call for help and provided critical surge capacity for patients and hospital systems in need. Truthfully, I can't say enough positive things about how Michigan Medicine responded to the acute crisis. We anticipate having a baseline census of patients with COVID-19 in the hospital for the next 18 months or so, in addition to possible further waves of illness to come.

From a departmental perspective, we also rapidly organized and mobilized with the realization that COVID-19 was about to have an enormous impact in our area. We are fortunate to have nearly 50 full-time clinical faculty in our urology department, so this afforded us some flexibility. Borrowing from the Lombardy model, we created three squads composed of faculty, trainees and advanced practice providers (for example, nurse practitioners) from all major urological disciplines (oncology, endourology, neuropelvic-reconstructive, general and paediatric) and deployed them on a schedule of 1 week in-person care followed by 2 weeks of virtual care. The intention was to limit health-care worker exposure as much as possible and to allow appropriate recovery time while providing all necessary care. We halted all elective cases across all specialties during the peak and performed only emergency and urgent procedures. From a urological perspective, this approach meant performing surgery for symptomatic bladder cancer, large renal tumours with caval involvement and urgent stone cases. Many of our residents and advanced practice providers volunteered for general and intensive care unit care responsibilities and some were deployed in this way. We were lucky that the crisis never reached a point that required all of us to be re-deployed, owing to the positive impact of social distancing in our area as well as the tremendous contributions of our medical, surgical and anaesthesia colleagues at Michigan Medicine. Since mid-May, we have begun the surgical ramping up process in a deliberate and thoughtful manner to optimize patient and provider safety.

Juan J. Andino. Our institution and department responded swiftly. Elective surgeries were cancelled, and leadership across the institution helped the transition from in-person to virtual encounters, reserving clinic space, emergency room and inpatient wards for patients with urgent or emergency issues.

Drs Palapattu, Kraft and Ambani (Urology Chair, Program Director and Assistant Program Director, respectively) immediately worked with the chief residents to design a schedule focused on safety, reducing the risk of potential exposures and minimizing the use of PPE. We were initially divided into three groups - one group would divide up tasks in the hospital whereas the remaining two-thirds of residents worked virtually from home.

Residents absorbed multiple roles, covering consultations and inpatient care 
across our three main hospitals. Initially, this workload was manageable owing to the drop in volume from cancelled elective surgeries as well as the ability to address many clinical queries with e-consults. Telehealth was adopted for the inpatient hospital setting and when a physical examination was not likely to alter medical decision-making, recommendations were provided after a conversation and review of laboratory results and imaging ${ }^{1}$. However, as additional urgent procedures were being scheduled with increased testing capacity and operating room (OR) safety protocols, a clinically active cohort composed of one-third of the residents was no longer sufficient. We then transitioned to a two-team system Blue and Maize - to enable more effective coverage of operating rooms, consultations and inpatient wards.

At this time, we are planning how to return to a new normal, in which residents go back to previously scheduled rotations with flexible coverage determined by clinical needs and OR availability. Masks are now required in all patient care areas and anyone entering the hospital is screened at every entrance for symptoms and given a mask if they don't already have one. More widespread testing is enabling patients to be tested before scheduled surgery. Between this and Governor Gretchen Whitmer's thoughtful approach and executive orders promoting social distancing, the numbers in the hospital have dropped from a peak of 229 on 16 April 2020 to 8 on 26 June 2020 .

\section{James M. Dupree. One of our first} changes was to limit the number of patients coming to see us in clinic. We started by postponing clinic appointments for patients who were deemed to be at high risk of developing complications should they contract COVID-19, for example, immunosuppressed or elderly patients. Within about a week, we started postponing

\section{The contributors}

Juan J. Andino is a third-year urology resident at the University of Michigan. He completed his undergraduate and medical degrees, and an MBA at the University of Michigan. Dr Andino is interested in telehealth and health policy and hopes to work at the intersection of these fields to optimize the delivery of urological care.

James M. Dupree is an Associate Professor of Urology at the University of Michigan. He completed his residency at Northwestern University and his fellowship in Male Reproductive Medicine and Surgery at Baylor College of Medicine. He also completed a Health Policy Fellowship with the American College of Surgeons. Dr Dupree specializes in the advanced treatment of male infertility, and his research focuses on male infertility and reproductive health policy. Dr Dupree is also the Ambulatory Care Clinical Chief for Urology at the University of Michigan.

Christian Fuglesang S. Jensen received his medical degree from the University of Copenhagen in 2015 and is currently enrolled as a PhD trainee working with male infertility at the Department of Urology, Herlev and Gentofte Hospital. Dr Jensen has previously worked at the Department of Urology, University of Michigan, performing research into andrology and male infertility. Dr Jensen serves as chair on the ESSM Scientific Sub-Committee for new technologies and sexual function and is a co-founder and member of the Core Unit of the CopMich Collaborative.

Ganesh S. Palapattu is the George F. and Sandy G. Valassis Professor and Chair of the Department of Urology at the University of Michigan. He attended the University of Texas at Austin where he earned a Bachelor of Arts degree in Humanities and then Baylor College of Medicine in Houston, Texas, where he earned his medical degree. Subsequently, Dr Palapattu completed his surgical internship, urology training and chief residency in urology at the David Geffen School of Medicine at UCLA followed by a laboratory research fellowship in Urologic Oncology at the Johns Hopkins Hospital Brady Urological Institute. His clinical interest is in the evaluation and management of men with prostate and kidney cancer.

Jens Sønksen received his medical degree from the University of Copenhagen in 1988 and earned his PhD and Doctor of Medical Science in 1995 and 2003, respectively. He is currently Professor of Urology at the University of Copenhagen and Head of the Urological Research Center and Section of Andrology, Herlev and Gentofte Hospital, Denmark. Dr Sønksen is currently serving as Adjunct Secretary General of the European Association of Urology and is a co-founder and member of the Core Unit of the CopMich Collaborative.

Daniela Wittmann received her BA Hons at Keele University, Keele, UK, her Master's in Social Work at Simmons College School of Social Work, Boston, MA and her PhD at Michigan State University. She is an Associate Professor in the Department of Urology and Adjunct Associate Professor at the School of Social Work at the University of Michigan. Dr Wittmann is a leading member of the Brandon Prostate Cancer Survivorship Program at the University of Michigan and serves as the Chair of the Mental Health Committee of the Sexual Medicine Society of North America. She is also a member of the Prostate Health Committee of the Urology Care Foundation.

more patients, based on the urgency of their medical problems. We performed a similar triage for surgical cases and started postponing all but the most urgent urological surgeries, but we never closed our clinics or operating rooms completely and always cared for patients with urgent or emergency needs.

As we neared the peak of the COVID-19 patient surge, we prepared for the possibility of exceeding our hospital's inpatient capacity. We planned to create a field hospital in a nearby university athletic facility to care for patients who were COVID-19-positive. The urology faculty were planning to staff this field hospital to provide general medical care for patients who were improving but were not yet ready for discharge home. Thankfully, the community's social distancing measures prevented the COVID-19 patient volume from ever exceeding our hospital's capacity and that field hospital never opened.

Jens Sønksen. The COVID-19 pandemic had immediate and profound consequences for the Danish Health system, including the Department of Urology at Herlev and Gentofte Hospital. Despite a well-functioning health-care system, no plan of action was prepared for a situation like this and the initial phase was filled with a lot of uncertainties and communication deficits between the government, health boards, hospital boards and department administration. Within a few days of Denmark's lockdown on 11 March 2020, all elective surgery and outpatient clinic visits were cancelled or postponed and, if possible, replaced by telecommunications for diagnosis and follow-up monitoring. From one day to the next, the department was completely restructured, with doctors and nurses sent to COVID-19 test centres and COVID-19 wards and regular urology wards redesignated as isolation wards in preparation for the expected rise in the number of COVID-19 cases in Denmark. Every individual worked hard to 'flatten the curve' and enable the health system to cope with increasing COVID-19 cases. On a daily level, doctors could no longer meet at morning conferences, all large meetings ( $>10$ persons) were cancelled and the teaching of medical students during clinical rotation was postponed, although virtual teaching programmes were soon established. We are now slowly opening and have restarted outpatient visits and elective surgery, whereas acute urology and cancer diagnostics and treatment have been ongoing during the entire period. 
0]

How did your institution respond to the COVID-19 pandemic?

Daniela Wittmann. There was an immediate move to clear the hospital to accommodate the COVID-19 patient influx. Urological surgeries were cancelled. I am a sex therapist in the prostate cancer survivorship programme and this meant that my sex therapy appointments with patients with prostate cancer post-op would diminish for a while. My clinic and my research office were closed and all work moved to virtual platforms. I have been working from home seeing patients in video visits and doing research online with meetings via Zoom. Both are quite manageable, although doing virtual sex therapy has its challenges. However, owing to the decreased clinical volume, I have had time to start a research project on patients' and physicians' responses to the delay in cancer care during the pandemic; I am glad to have a chance to engage with this difficult situation in a meaningful way.

J.M.D. I was impressed with the speed and flexibility of our institution's response to the COVID-19 pandemic. Several weeks before the first case in Michigan, we created a centralized command centre to oversee institution-wide clinical operations. Once COVID-19 reached our state, we contracted our clinical sites, triaged our surgical cases and doubled our intensive care unit capacity. Many of these changes were enabled by our employment structure: the physicians, nurses, nurse practitioners, physician assistants and all the other members of the clinical workforce are employees of the institution, which helped us to respond in a coordinated fashion.

In addition, our medical group had recently implemented new layers of clinical leadership - including physicians, administrators and nurses - to oversee our clinics. These clinical leaders were instrumental in our response. There were instances of miscommunication or challenges with inconsistent decision-making, but these instances were rare, and the benefits of the clinical leadership triads were immense.

Christian Fugelsang S. Jensen. Denmark closed down on the evening of Wednesday 11 March 2020. Listening to the press briefing from the Prime Minister I realized how big an impact the COVID-19 pandemic would have on clinical research. The next day I could not go to work at the Urological Research Unit at Herlev and Gentofte

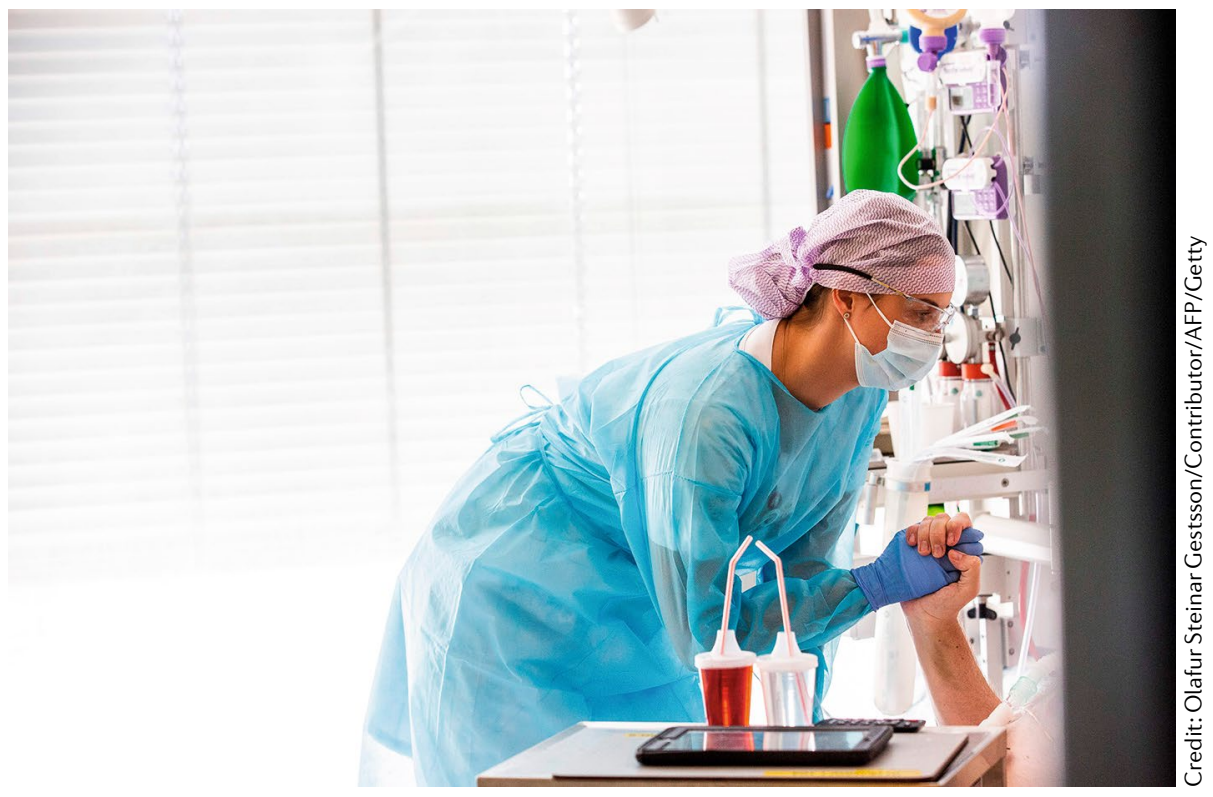

Hospital and had to cancel all my patients' visits and elective surgeries. I was in the final months of completing my $\mathrm{PhD}$, a randomized surgical trial on sperm retrieval in men with non-obstructive azoospermia, but had to pause all related clinical activities. As part of my PhD, Professor Dana Ohl from the University of Michigan performs surgery with me on all study patients in Denmark, but the travel ban made this impossible. Currently, the travel ban is still in place, but we are working on possibilities for finishing the study and the $\mathrm{PhD}$.

J.S. As Chair of Urology at the University of Copenhagen it was my responsibility to find alternatives for teaching medical students. We successfully established e-learning platforms with interactive sessions for discussion and we had to restrict clinical teaching at hospitals to limit the possible spread of COVID-19. For students on clinical rotations who normally have a real patient as part of their clinical urology examination, we replaced the patient with a urologist in the role of a patient and could then complete the exams.

0] What did your institution do, formally and informally, to provide support for faculty and staff during the COVID-19 pandemic?

\section{G.S.P. The COVID-19 crisis revealed} the true character of our department: compassionate and selfless. People looked out for one another and sought ways to provide support to those in need. One of our faculty in paediatric urology, Dr Courtney Streur, created the Daily (Uro)Flow, a spontaneous and voluntary daily email from a department member describing how they were coping with the crisis, often with funny anecdotes. Plenty of Zoom calls were had to maintain connectivity and provide updates. I know many faculty and staff who reached out to each other during the peak to lend support and check on each other. Our residency programme director, Dr Kate Kraft, met with all of our residents weekly via Zoom to check in and we maintained our weekly department conference schedule via video conference. As much as possible, we tried to remain connected.

\section{J.M.D. I think everyone on our team} felt scared, anxious and/or frustrated at various times during the pandemic. It was an unprecedented time. Our institution advertised counselling services that all faculty or staff could use. I took advantage of these counselling services in the heat of the pandemic and found them invaluable for reinforcing my ability to help to lead our department's operational response. Living and working through this pandemic were powerful reminders that we need to take care of ourselves so that we can take care of others.

D.W. A number of supportive activities were organized by Michigan Medicine, including counselling for staff and faculty and information websites. Hospital system leadership provided daily updates about hospital census of patients with COVID-19, employee testing and infections. Weekly Town Halls were held for all employees with information about state and hospital statistics, workforce deployment, 


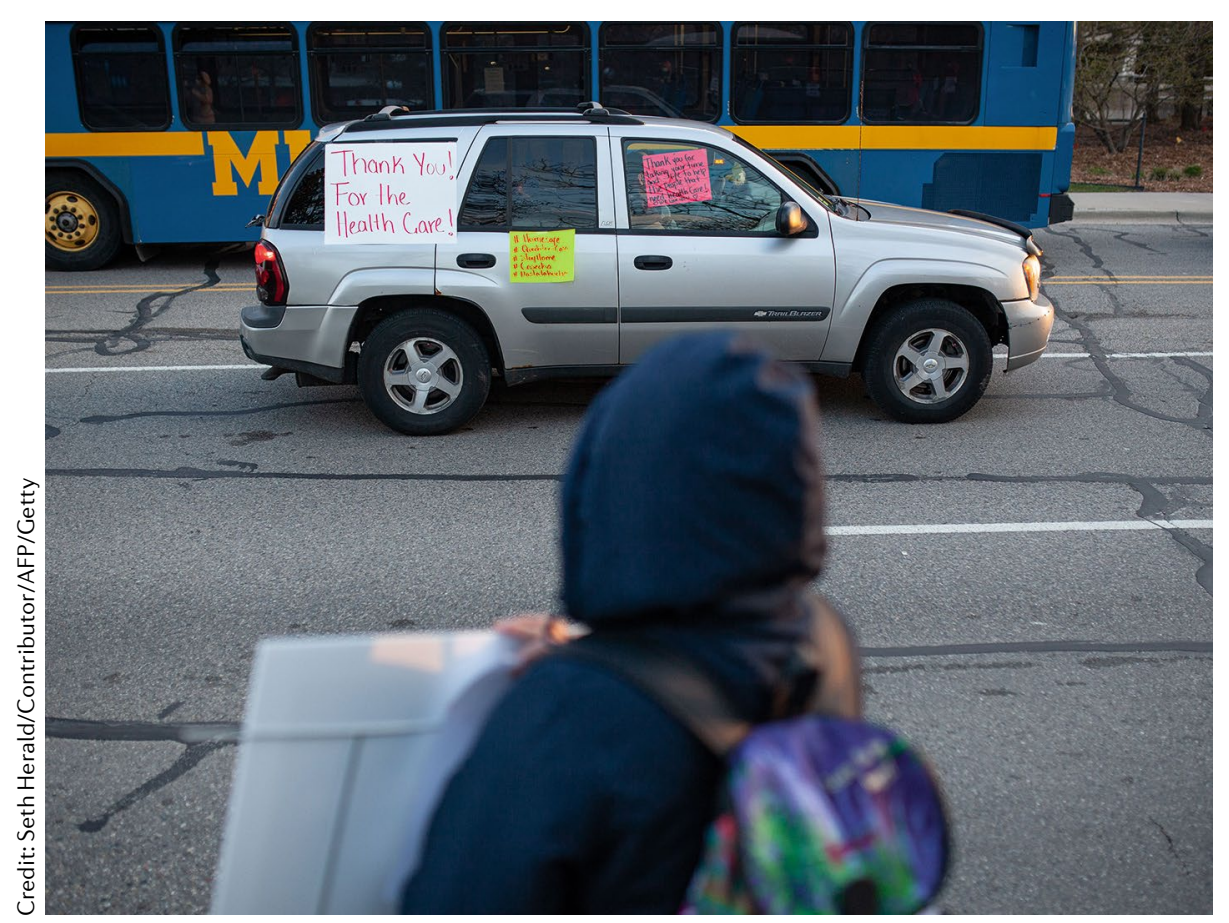

financial impact of the pandemic on the institution and Q and A opportunities. In the Department of Urology, the Chair provided weekly updates on issues relevant to the faculty, such as plans for potential deployment in the COVID-19 zones, organization of urology services, team organization with health-protective strategies, OR availability and financial impact of the pandemic. The departmental Wellness Committee invited faculty and staff to a Facebook page to share experiences, both triumphs and challenges. The Facebook page also posted resources, such as where to get food deliveries or how to talk to children about COVID-19. A faculty member began an informal All Staff and All Faculty email exchange, “The Daily (Uro) Flow”, in which individual staff or faculty members posted narratives and photos about how they and their families were living and coping during the pandemic. In all these initiatives and activities, emphasis was placed on viewing Michigan Medicine and the Department of Urology as a family that would support each other, get through the pandemic together and become strong as a result.

J.S. On a continuing basis, the hospital administration and our department have sent out frequent COVID-19 email newsletters to all staff members, including a summary of information from health authorities to support the dissemination of important information. Throughout the entire period, everyone has received full salaries, including those who were sent home without the possibility of continuing to work. Finally, all health-care workers have experienced huge support from society in general, with positive news coverage of the efforts of health workers and with examples including occasional free meals and snacks for staff on duty, free car rental for health-care workers in Copenhagen and free isolation stays at hotels for infected individuals.

\section{What will be the long-term effects of COVID-19 in your institution?}

J.J.A. The pandemic has expanded the use of telehealth owing to rapid and wide-spread changes in national and state-specific regulatory and licensing policies. Even beyond the pandemic, many more patients will have the option of following up with their doctors through video visits ${ }^{2}$. Ideally, this will keep healthier patients at home and open up capacity for patients with acute issues and complex medical needs. Hopefully, this will also give providers additional flexibility and more control over where and how they care for their patients.

This period will also have a lasting impact on our approach to medical education. We have seen how online sessions can reach a broad audience and that videos can be recorded and stored for review at a later time. In the future, medical schools might not need to continue growing in size if the majority of their content can be distributed electronically. Space can be shared and used for specific, hands-on tasks such as cadaver labs, simulations and selected group activities. Similarly, future medical students will be introduced to telehealth as a part of their curriculum. They will start performing video visits at the same time as learning how to do a physical examination in the clinic. Finally, this pandemic might even change the entire interview process; it is not impossible to imagine a system whereby applications are narrowed down through initial review, then virtual interviews and ending with a more selective pool of applicants interviewing in person. It will be interesting to see what long-term changes will come from COVID-19 that we cannot even foresee.

It is also clear that not everything can be shifted to a virtual medium. A different kind of fatigue comes from being connected to a computer or device for most of the day. I miss the sometimes brief but meaningful interactions that take place in the hospital, conference room and lecture halls. A quick hello, swapping of stories, a pat on the back from a colleague you haven't seen in a while. These are all lost when people are bouncing online through innumerable Zoom sessions.

D.W. I expect that there will be more video visits in usual clinical care in the future, as long as insurance coverage continues. I also expect that Michigan Medicine will remain very much prepared to handle a possible fall spike in COVID-19 and that fast reorganization might occur again. It is not clear when in-person visits for usual care will return, given the lack of testing and treatment for COVID-19. Some patients are already voicing their preference for virtual care, citing both convenience and fear of becoming infected.

J.M.D. I am confident that the principal long-term effect will be the expanded use of telehealth ${ }^{3}$. We were using some telehealth before the COVID-19 pandemic, but when we had to postpone patients from in-person care, telehealth became essential. Even now, as patients are returning to our clinics, the benefits of telehealth - for doctors and for patients - remain.

I also expect us to restructure our clinical work. When we shut down all but the most urgent urological care, it gave us the opportunity to rebuild our clinical delivery system from the ground up, addressing longstanding barriers and reimagining how we can best provide high-quality, safe and efficient urological care to patients. 
Finally, I expect, for better or worse, that there will be more centralized control of clinical operations in our institution. Centralized control was necessary during the pandemic to have a uniform and coordinated response. It will be hard to unwind some of that centralization. However, I think that individual clinics will need to regain some of their autonomy to help facilitate innovation and adapt to the unique needs of local patient populations.

G.S.P. There likely will be many long-term effects of COVID-19 at Michigan Medicine. Virtual care is one. Additionally, extended clinic hours ( $7 \mathrm{am}-7 \mathrm{pm})$ and the addition of Saturday morning clinics and routine Saturday scheduled surgeries, staffed on a rotating basis, will also be likely incorporated on a long-term basis. The immediate financial impact has forced us to make some hard decisions regarding programmes and personnel. I suspect that in the future we will be much more cost conscious, which is not necessarily a bad thing. I also firmly believe that we as a department and institution will be better and stronger on the other side of COVID. This crisis has required us to take a hard look at what we do and how we do it and, importantly, made us prioritize and economize. Although hard to envision at the moment, I strongly feel that we will be in a better position 1 year from now than we were 1 year ago to take care of patients, make discoveries and educate the leaders of tomorrow.

C.F.S.J. Aside from constant hand hygiene, physical distance and mandatory SARS-CoV-2 tests for health-care personnel and patients, I foresee less physical interaction in general, including meetings, conferences and travel. Although virtual platforms can replace many of these activities, I fear that valuable aspects of direct interaction will disappear, leading to reduced sharing of information and experiences, as well as decreased input from peers. The lack of direct networking may even lead to a reduction in collaboration between institutions and a decrease in the development of new projects to benefit future patients. This has direct implications for programmes such as CopMich (The Copenhagen Michigan Urological Collaboration), which is an example of a collaboration built on direct interaction between Professor Jens Sønksen and Professor Dana A. Ohl (Department of Urology, University of Michigan), both clinical researchers sharing similar research interests. Without the established friendship, CopMich would not have been as successful as it has been, with more than 65 shared publications over 25 years. I am fortunate to be part of this friendship and I truly see the personal and scientific value in the relationships created ${ }^{4}$.

J.S. As a consequence of cancelling and postponing appointments for patients with urological conditions, there is a build-up of patients needing evaluation and treatment, which will require a substantial amount of time and effort to catch up on. This backlog will have implications for the workload of our staff and will limit the possibility for participating in educational and research-related activities, including participation in conferences. In a public-health system, we cannot expect additional financial resources for catching up, especially given the enormous amount of money used on governmental emergency relief plans. As a result, I fear we will lack money and opportunities to continue educational activities as we used to before the COVID-19 pandemic.

What lessons were learned during the response to COVID-19?

D.W. I have learned that I can do a lot of my work remotely. I have also learned that much of the ability to work effectively with others in this way is due to being able to rely on the comfort of pre-existing relationships. Doing new patient assessments or interacting with new research colleagues only virtually misses important human dimensions. I miss my colleagues and in-person patient care. As a health-care provider, I have also felt powerless, not useful, given the overwhelming need for specific expertise that I did not have. I offered to volunteer for blood donation and mental health services. I worried about providers on the front line of the COVID-19 work. I was reminded of how important it is to me to be of service.

J.J.A. I've had two main takeaways: first, the importance of your colleagues, co-workers and leaders in the workplace. In the face of an unprecedented pandemic and the anxiety evoked when models predicted that our hospital would be overrun within a matter of weeks, people were flexible and willing to adapt to work as part of a team - taking on tasks that would normally be split between two or three people, covering the on-call pager at night and on weekends that were previously scheduled for someone else. I feel very fortunate to be a part of this group.
Residency itself is a shared experience like few others; add a pandemic to the mix and this is something that will connect us for the rest of our lives. Second, we have had the technology to connect with friends and family who live far away and have underused it for a long time. For the first time in years, I've had long phone and FaceTime conversations with people all around the country and world. Rather than texting or emailing, I've picked up my phone or used my laptop to talk, watch concerts together, and celebrate or mourn together.

G.S.P. I think there are several lessons we have learned from COVID-19 thus far. First, we are at our best when we work together. The collaborative spirit shown by our team during this time of crisis is inspiring. Second, we have seen the importance of communication. Frequent and clear communication from leadership about the status of the epidemic and our response to it, as well as the consequences of the crisis (such as financial issues), engendered better understanding of what was happening and built trust. Third, virtual care really does work! Our department had been using various methods of virtual care before the pandemic, but over the past 2 months our usage went up dramatically. For the most part, patients and providers have found virtual care convenient and I anticipate that it will play a major role in how we care for patients moving forward ${ }^{1}$.

J.M.D. I believe we will be realizing lessons from the COVID-19 pandemic for years to come. My most important lesson was a reminder about the importance of institutional culture. As I described above, our institution completely reorganized our clinical care operations within a few days. Everyone's schedule changed, which, as you can imagine, was very disruptive. Thankfully, our urology department has a culture that values collaboration, teamwork and a shared sense of responsibility for our patients. These qualities shone as we responded to the daily changes in our lives.

Personally, I also learned several lessons about leadership. First, communication is a cornerstone of leadership, especially in a crisis. We used regular, clear and frank communication to reorganize our workforce and adapt to the changing epidemiology of the pandemic. Second, I learned the importance of being vulnerable and admitting when I do not know what is going to happen next. However, I also learned that it is valuable to pair that vulnerability 
with a clearly articulated belief in our ability to get through the pandemic together. Third, I was reminded about the benefits of feedback. It is tempting for leaders to make their own plans, assuming they know what is best. However, each time I asked for feedback on my plans, especially feedback from those on the front lines such as nurses, medical assistants and administrators, their responses made the plans better. There truly is wisdom in the crowd. Finally, I was reminded to give others the benefit of the doubt. In stressful times, there will inevitably be interpersonal conflict. Believing that everyone is trying their best given the circumstances was helpful as I navigated those conflicts.

C.F.S.J. I have learned to appreciate going to work as I quickly realized that working from home had several downsides and limitations and completely lacked the social aspect and daily input from colleagues. On the other hand, I learned that many activities, including meetings, do not necessarily have to be physical but can often be replaced by virtual meetings. However, this approach requires discipline and is a skill that needs to be learned ${ }^{2}$.

J.S. Many lessons can be learned from this unforeseeable situation. The most important thing is to have a plan for such situations.

This was seemingly neglected, as Denmark has not been in a real emergency since World War 2. Furthermore, a clear structure for communication should be in place to avoid misinformation and misinterpretation.

Juan J. Andino ${ }^{1 凶}$, James M. Dupree (D) ${ }^{1 凶}$, Christian F. S. Jensen ${ }^{2}$, Ganesh S. Palapattu $^{1 凶}$ Jens Sønksen ${ }^{2}$ and Daniela Wittmann (iD) ${ }^{\text {凶 }}$

${ }^{1}$ Department of Urology, University of Michigan, Ann Arbor, MI, USA.

${ }^{2}$ Department of Urology, University of Copenhagen, Herlev and Gentofte Hospital, Copenhagen, Denmark.

凶e-mail: juanjose@med.umich.edu; jmdupree@med. umich.edu; fullejensen@gmail.com; gpalapat@med. umich.edu; jens@sonksen.dk; dwittman@med.umich.edu

https://doi.org/10.1038/s41585-020-0352-6

Published online 6 July 2020
1. Gadzinski, A. J. et al. Telemedicine and eConsults for hospitalized patients during COVID-19. Urology https://doi.org/10.1016/j.urology.2020.04.061 (2020)

2. Gadzinski, A. J. \& Ellimoottil, C. Telehealth in urology after the COVID-19 pandemic. Nat. Rev. Urol. https:// doi.org/10.1038/s41585-020-0336-6 (2020).

3. Ellimoottil, C. What will urology look like in 2035 ? Nat. Rev. Urol. 16, 635-636 (2019).

4. [No authors listed] 15 years of urology at Nature: a celebration of collaboration. Nat. Rev. Urol. 16, 631 (2019).

Acknowledgements

This work was carried out on behalf of the CopMich Collaborative group. The authors from the University of Michigan and Herlev and Gentofte Hospital wish to acknowledge and thank all the nurses, physicians, nurse practitioners, physician assistants, medical assistants, students, schedulers, call centre agents, office staff and clinical staff who rose to the occasion and cared for our patients. More than any other time, responding to the COVID-19 pandemic was a team effort.

\section{Competing interests}

J.M.D. declares grant funding from Blue Cross Blue Shield of Michigan for quality improvement activities. The other authors declare no competing interests.

\section{Publisher's note}

Springer Nature remains neutral with regard to jurisdictional claims in published maps and institutional affiliations.

\section{RELATED LINKS}

The Copenhagen Michigan Urological Collaboration: https://www.copmich.com/

(c) Springer Nature Limited 2020 